\title{
Person Re-Identification by Common-Near-Neighbor Analysis
}

\author{
Wei LI ${ }^{\dagger * a)}$, Nonmember, Masayuki MUKUNOKI ${ }^{\dagger \dagger}$, Member, Yinghui KUANG ${ }^{\dagger \dagger}$, Yang $\mathrm{WU}^{\dagger \dagger}$, Nonmembers, \\ and Michihiko MINOH ${ }^{\dagger \dagger}$, Fellow
}

\begin{abstract}
SUMMARY Re-identifying the same person in different images is a distinct challenge for visual surveillance systems. Building an accurate correspondence between highly variable images requires a suitable dissimilarity measure. To date, most existing measures have used adapted distance based on a learned metric. Unfortunately, real-world human image data, which tends to show large intra-class variations and small inter-class differences, continues to prevent these measures from achieving satisfactory re-identification performance. Recognizing neighboring distribution can provide additional useful information to help tackle the deviation of the to-be-measured samples, we propose a novel dissimilarity measure from the neighborhood-wise relative information perspective, which can deliver the effectiveness of those well-distributed samples to the badly-distributed samples to make intra-class dissimilarities smaller than inter-class dissimilarities, in a learned discriminative space. The effectiveness of this method is demonstrated by explanation and experimentation.

key words: person re-identification, common-near-neighbor analysis, learned metric, visual surveillance
\end{abstract}

\section{Introduction}

\subsection{Background and Related Work}

Person re-identification presents a unique challenge to modern video surveillance as it involves a number of hard problems: large intra-class variations and small inter-class differences caused by pose change, illumination alteration, viewpoint shift, occlusion, body shape resemblance, clothing style similarity, and so on [1].

Current approaches to person re-identification can be divided into two paradigms. The first focuses on robust feature representation of personal appearance information. This paradigm is loosely related to our proposed method, and helps us to understand the development of this issue. There are several noteworthy examples of this paradigm. For example, Haar-like feature and DCD (Dominant Color Descriptor) [2] equipped with the AdaBoost scheme can form an invariant and discriminative signature of a person,

Manuscript received April 3, 2014.

Manuscript revised July 17, 2014.

The author is with the Department of Intelligence Science and Technology, Graduate School of Informatics, Kyoto University, Kyoto-shi, 606-8501 Japan.

${ }^{\dagger}$ The authors are with Academic Center for Computing and Media Studies, Kyoto University, Kyoto-shi, 606-8501 Japan.

${ }^{\dagger \dagger}$ The author is with Chien-Shiung Wu College, Southeast University, Nanjing, 210096, China.

*Presently, with the Institute of Scientific and Industrial Research, Osaka University, Ibaraki-shi, 567-0047 Japan.

a) E-mail: seuliwei@126.com

DOI: 10.1587/transinf.2014EDP7102 whereas HPE (Histogram Plus Epitome) [3] focuses on general chromatic content and recurrent local patches to extract the complementary global and local features of human appearance. SDALF (Symmetry-Driven Accumulation of Local Features) [4], [5] accumulates three types of local features regarding the symmetric and asymmetric property of body structure. MRCG (Mean Riemannian Covariance Grid) [6] uses grid based covariance descriptors to capture the textual information within local patches of a human body. USL (Unsupervised Salience Learning) [7] applies adjacency constrained patch matching to build dense correspondence between image pairs to handle viewpoint and pose variations, and it exploits human salience in an unsupervised manner to search for discriminative and reliable patch matches.

The second paradigm relies on suitable dissimilarity measurements after feature representation. Our proposed method belongs to this paradigm. Since, in the feature space, images of people can be regarded as sample points, a suitably-crafted measure can improve their intra-class compactness as well as inter-class separation, allowing stronger discrimination [8]. For instance, LMNN (Large Margin Nearest Neighbor) [9], [10] learns a metric by maximizing the margin to distinguish the samples from different classes. By contrast, RDC (Relative Distance Comparison) [11] is formulated to maximize the likelihood that a pair of true matches will have a smaller distance than a wrong match pair, making it more tolerant of appearance changes and less susceptible to model over-fitting. RankSVM [12] translates the problem from one of absolute scoring to one of relative ranking, and learns a metric space in which the highest ranking pair, rather than the closest by direct distance measure, is treated as a potential match. KISSME (Keep It Simple and Straightforward MEtric) [13] uses an effective and efficient strategy to learn the distance metric based on equivalence constraints from a statistical inference perspective. LAFTaV (Locally Aligned Feature Transforms across Views) [14] assigns images to different local experts according to the similarity of their cross-view transforms before projecting them into a common feature space and matching them with a locally learned discriminative metric.

Since human image data in a feature space can be treated as points, their correspondences can be determined by the dissimilarity score; therefore, a suitable dissimilarity measurement becomes fairly important. So far, in literature, there have been plentiful distances that directly and 
solely concentrate on the pair of points in a pre-defined metric space. Although, this kind of classical distance is discriminative sometimes, yet it tends to be incapacitated by the complex real-scenario human image data of large within-class variations and small between-class differences in general. Accordingly, to accurately re-identify the human image data, we creatively propose to encapsulate the neighborhood-wise relative information into a novel dissimilarity in a discriminative space, as a new trial of neighborhood dependent dissimilarity measurements. Hereinafter, the neighborhood structure indicates the spatial layout relationship between the neighboring samples and the concerned sample.

Two recent methods of the second paradigm are closely related to our proposed approach. They have achieved encouraging results in the fields of person re-identification and facial tagging, respectively. OMRR (Optimizing Mean Reciprocal Ranking) [15] searches for a suitable metric space to separate human image classes based on Euclidean distance. This method optimizes the list-wise ranking through the design of the loss function of MLR (Metric Learning to Rank) [16]. OMRR has achieved better results than previous approaches, but real-world challenges of the serious visual difficulties and/or limited sample size more or less affect its performance, so many of the human image data in the learned metric space are still inseparable based on the traditional Euclidean distance. To overcome the weakness of this method in discriminating real-world human image data, we initially propose to quantify the situation of the local neighborhood structure of one sample in the neighborhood structure of the other sample into a dissimilarity in the learned metric space. To show the advantage of our proposal, Rank-Order distance [17] provides a good contrast. Rank-Order distance uses rank order quantization to solve the samples' non-uniform distribution problem during clustering. Though this method quantizes the neighborhood structure information comparison, it only works well on facial tagging, and is incompetent for the challenging problem of person re-identification that includes more intractable real-world complexities.

\subsection{Overview of Our Approach}

For re-identification, the people in one camera usually have their corresponding matches in the other camera, and this ground-truth assumes the nonoccurrence of turn-back cases. This study copes with the situation of re-identifying the query human image from the corpus side, and, more concretely, there is one image for each person on the corpus side, and one or possible more images for him/her to be reidentified on the query side.

To this end, we creatively reformulate this problem as finding the correct human image match from the query side to the corpus side, and address the novelty of using the side information, which means the remaining images from both query and corpus sides besides the measured image pair. In practise, this reformulation can help re-identify the incom- ing and outgoing pedestrians between the entrance and exit of the shopping mall, airport, and so forth. Correspondence in some camera network can also be established if human images from all the entrances and exits are collected, respectively. Every time, when we match the query image in question to the corpus side, we let those remaining images participate to help matching. These unlabeled image data potentially contains the neighborhood information in the feature space for each identity in the corpus, but their usefulness hasn't been considered in many previous works. We expect such additional useful information to be an effective weapon against the challenge of intra-class variations and small inter-class differences in re-identification. Motivated by the expectation of delivering the effectiveness of those welldistributed samples to the badly-distributed samples during measure, we propose the CNNA (Common-Near-Neighbor Analysis) method, which designs the CNNM (CommonNear-Neighbor Modeling) dissimilarity in a metric space of improved intra-class compactness and inter-class separation learned by OMRR.

This paper is based on our international conference papers [18] and [15], with the addition of in-depth theoretical analysis and explicit experimental testing.

\section{Common-Near-Neighbor Analysis}

\subsection{Common-Near-Neighbor Modeling}

\subsubsection{Idea of CNNM}

Person re-identification is recast to the problem of discriminating human image data based on measuring the distances among samples in feature space. When the feature space has large intra-class variations and small inter-class differences, isolated to-be-measured sample pair may make traditional point-wise distance lose effect. To address this, we reconsider the distance measure from the neighborhood-wise relative information perspective, and propose a novel dissimilarity, as a new trial to overcome the vulnerability of traditional point-wise distance.

Let us begin with a simple re-identification example. Usually, due to large intra-class variations and small interclass differences, traditional distance, like L1-norm, L2norm, will lose effect to some extent when building the correspondences between the pair of query and corpus samples, as illustrated in Fig. 1. In this figure, samples classes can be distinguished by colors and shapes. When $c$ is selected as a query, $b$ and $d$ are assigned in the corpus side. $c d$ are badlydistributed, while $a b$ and $e f$ are well-distributed. Clearly, by Euclidean distance, the sample $b$ will rank before the sample $d$ with regard to the sample $c$. To solve this problem, we suggest borrowing the effectiveness from those well-distributed samples to improve the badly-distributed samples. To this end, we propose to quantify the local neighborhood structure of the paired samples in each other's neighborhood structure, and model the quantity of the common nearness of the local neighborhood structure as a dissimilarity. We name 


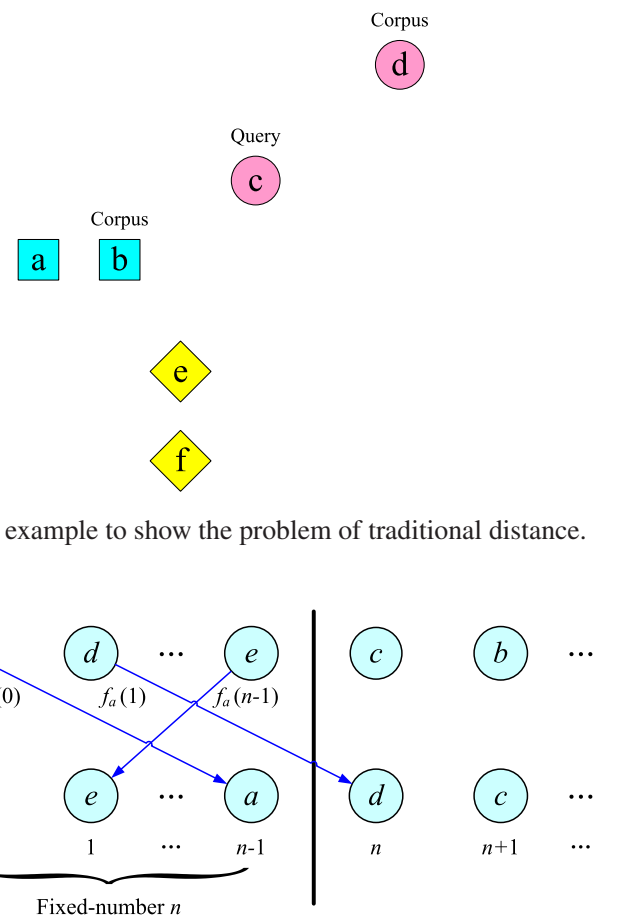

Fig. 2 Illustration for "Symmetric dissimilarity" of CNNM. $D_{a}^{\text {Fixed-number }}$ (b) is calculated from the $0^{\text {th }}$ to the $(n-1)^{\text {th }}$ nearest neighbor in $a$-list, and $D_{b}^{\text {Fixed-number }}(a)$ is calculated in a similar way.

this dissimilarity from the semantic perspective, "CommonNear-Neighbor Modeling".

\subsubsection{Formulation of CNNM}

Neighborhood structure can be described and compressed by the rank order lists in terms of farness and nearness. Considering the feature space having the class structure, we recommend a moderate and fixed local neighborhood to incorporate the within-class sample distribution information. Technically, we suggest a "Fixed-number" $n$ instead of a flexible number on the top several samples of the rank order lists, and count the top fixed-number elements of one list in the other list symmetrically as "Symmetric dissimilarity", which is given by:

$$
D^{\text {Symmetric }}(a, b)=D_{a}^{\text {Fixed-number }}(b)+D_{b}^{\text {Fixed-number }}(a),
$$

where

$$
D_{a}^{\text {Fixed-number }}(b)=\sum_{i=0}^{n-1} O_{b}\left(f_{a}(i)\right) .
$$

In Eqs. (1) and (2), $a$ and $b$ are a pair of to-be-measured samples. $f_{a}(i)$ returns the $i^{\text {th }}$ element in $a$-list, and $O_{a}(b)$ is the rank order of $b$ in $a$-list. $O_{b}\left(f_{a}(i)\right)$ is the rank order of $f_{a}(i)$ in $b$-list. $D_{a}^{\text {Fixed-number }}(b)$ is calculated by summing the rank orders of the top fixed-number elements of $a$-list in $b$ list, as shown in Fig. 2. $D_{b}^{\text {Fixed-number }}(a)$ is calculated similarly. Here, rank order lists are formed by sorting Euclidean distances between the measured samples and all the other samples.

Since the "Fixed-number" value can be crucial to the performance of "Symmetric dissimilarity", it deserves further discussion. If the neighborhood is too large, intruder samples from different classes may disturb the encoding of representative locality for the sample. In this case, the "Symmetric dissimilarity" of samples from different classes will have tendency to be lower than that of samples from the same class. If, on the other hand, the neighborhood is too small, local information of the outlier samples may not be sufficient and capable enough to counteract the variations. In this case, the "Symmetric dissimilarity" of samples from the same class will have tendency to be higher than that of samples from different classes. Obviously, both cases will negatively affect ranking/classifying performance, and should be avoided. For a properly discriminative "Symmetric dissimilarity", we suggest using a "Fixed-number" $n$ set to approximately $N / 2$ of tradeoffs (where $N$ is the approximate average sample number per person known beforehand, or estimated from dividing the total image number by the identity number) to avoid the cases that the neighborhood is either too large or too small. Note that this suggestion is based on observation and could not be demonstrated through mathematical proof.

In particular, for the single-shot vs. single-shot reidentification problem, there are only two samples for each person and the recommended "Fixed-number" will be " $n=$ 1". Then, each sample has to consider the situation of itself within the neighborhood structure of the other sample. In this case, the asymmetric ranking problem is rather obvious. Typically, a given pair of samples will not yield the same rank order for each other in their own rank order lists. This asymmetric ranking problem originates from the deviation of the isolated samples in the space of large intra-class variations and small inter-class differences. Since it would be heuristic and unfair to judge rank order by randomly selecting one of these ranks, or simply averaging the two, we turn to the added discriminative ability of "Asymmetric dissimilarity", as given by:

$$
D^{\text {Asymmetric }}(a, b)=\min \left(O_{a}(b), O_{b}(a)\right) .
$$

Joining "Symmetric dissimilarity" and "Asymmetric dissimilarity", we present a new dissimilarity modeling, CNNM, as given by:

$$
\begin{array}{r}
D^{\mathrm{CNNM}}(a, b)=D^{\text {Symmetric }}(a, b) \\
+2 \lambda n D^{\text {Asymmetric }}(a, b),
\end{array}
$$

where $\lambda$ is a trade-off parameter to balance "Symmetric dissimilarity" and "Asymmetric dissimilarity". This makes the model more compatible. Whereas "Symmetric dissimilarity" uses the "Fixed-number" of the rank orders in consideration of symmetry, "Asymmetric dissimilarity" uses only one rank order. Thus, it is reasonable to reformulate these two dissimilarities by doubling $n(2 n)$ and thereby unifying dimensions. 
For the example of Fig. 1, b-list is baecfd, $c$-list is cbdeaf, and $d$-list is dcbeaf. Since rank orders are defined as 012345 , the CNNM dissimilarities will be $D^{\mathrm{CNNM}}(b, c)=$ $1+3+2 \times 1 \times 1=6 ; D^{\mathrm{CNNM}}(c, d)=1+2+2 \times 1 \times 1=5$ by setting $n=1$ and $\lambda=1$ ( $\lambda$ selection will be explained latter). Thus, $d$ will rank before $b$ with regard to $c$ by this dissimilarity measure. Analytically, let us check the rank order lists for $b, c$, and $d$. When comparing $b$-list and $c$-list, we can find that, because $a$ and $b$ are close, $a$ rank before $c$ in $b$ list. This leads up to the increase of the inter-class distance $D^{\mathrm{CNNM}}(b, c)$. Moreover, when comparing c-list and d-list, we can find that, because $e$ and $f$ are far away from $c$, they stably reside in the tails of $c$-list and $d$-list. This contributes to the decrease of intra-class distance $D^{\mathrm{CNNM}}(d, c)$. Actually, for $c$ and $d$, CNNM considers how $c$ stays in the neighborhood structure of $d$, and how $d$ resides in the neighborhood structure of $c$, simultaneously. If $c$ is the near neighbor to $d$, and $d$ is the near neighbor to $c$, we note this situation $c$ and $d$ are "common-near" neighbors to each other. This common-near-neighbor information can be measured by the CNNM dissimilarity.

There are some noteworthy aspects on this dissimilarity as well. As the precondition, there needs to exist some welldistributed samples in the feature space. This guarantees the precondition for effective CNNM measure. Actually, this requirement is easy to satisfy in a carefully designed or selected feature space. As the limitation, though the welldistributed samples can help those badly-distributed samples utilizing CNNM, the amount of and the power of the well-distributed samples in the feature space are limited, thus only those badly-distributed samples whose situation is not rather serious can be ameliorated. And, here, we clarify the phrase "not rather serious" by the specific cases. If the pair of elements to be measured in the same class stay quite farther apart than other samples from different classes, or intruded by different-class samples lying between them, CNNM dissimilarity tends to lose efficacy.

\subsection{Comparison between CNNM and Its Analogue}

\subsubsection{Weakness of Rank-Order Distance}

There exists one similar method, Zhu et al.'s Rank-Order distance, originally designed for clustering during face tagging [17]. Although this method is capable of solving the samples' non-uniform distribution problem during clustering by rank order quantization, yet it may fail to re-identify the human image data with large intra-class variations and small inter-class differences, as the general case in the real world. Note that, in Zhu et al.'s work, clustering addresses the absolute threshold for filtering the distances, while in this paper re-identification emphasizes the relative comparison between the intra- and inter-class distances. Clustering and re-identification are different in nature, so we should not impose Rank-Order distance on re-identification.

Zhu et al.'s Rank-Order distance is given by:

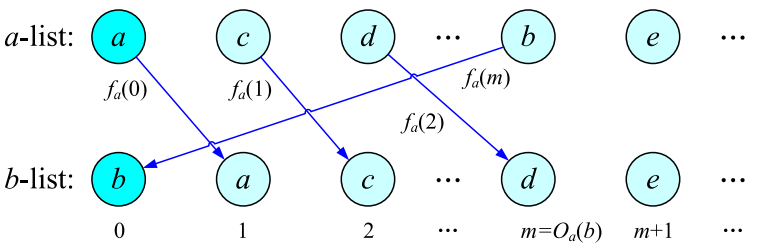

Fig. $3 a$-list and $b$-list are rank order lists. $D_{a}(b)$ is calculated from the $0^{\text {th }}$ element to $b$ in $a$-list, while $D_{b}(a)$ is calculated from the $0^{\text {th }}$ element to $a$ in $b$-list. Typically, $D_{a}(b) \neq D_{b}(a)$. Note that, here, for conciseness and comprehensibility, only $O_{b}\left(f_{a}(i)\right)$ are visualized by arrows in this figure. Each arrow head points the rank order $O_{b}\left(f_{a}(i)\right)$ in $b$-list for the element $f_{a}(i)$ in $a$-list that connects the arrow tail.

$$
D^{\text {Rank-Order }}(a, b)=\frac{D_{a}(b)+D_{b}(a)}{\min \left(O_{a}(b), O_{b}(a)\right)}
$$

where

$$
D_{a}(b)=\sum_{i=0}^{O_{a}(b)} O_{b}\left(f_{a}(i)\right)
$$

In Eqs. (5) and (6), $a$ and $b$ are a pair of samples to be measured. $f_{a}(i)$ returns the $i^{\text {th }}$ element in $a$-list, and $O_{a}(b)$ is the rank order of $b$ in $a$-list. $O_{b}\left(f_{a}(i)\right)$ is the rank order of $f_{a}(i)$ in $b$-list. $D_{a}(b)$ is calculated by summing the rank orders of the top several elements of $a$-list in $b$-list, as shown in Fig. 3. $D_{b}(a)$ is calculated in a similar way. Here, rank order lists are generated by sorting Euclidean distances between the measured samples and all the other samples.

Despite robustness to clustering, this dissimilarity doesn't consider the potential class information, so it will be non-discriminative for the sample space of the class structure, like in the re-identification situation. From the formulation level, the weakness comes from the flexible neighborhood size and the inappropriate denominator. Evidently, Eq. (5) is sensitive to $O_{a}(b)$ and $O_{b}(a)$, which reflect the position relationship between paired samples $a$ and $b$. If $O_{a}(b)$ and $O_{b}(a)$ are too big or small, the local neighborhood structure of each sample cannot sufficiently represent the class information, thus the comparison will have no discriminatory power. If $O_{a}(b)$ and $O_{b}(a)$ are quite different, the local neighborhood structures for each sample pair become incomparable, and worse still, the biased denominator will deteriorate the normalization.

\subsubsection{Advantage of CNNM}

In addition to analysis, here, we further illustrate the advantage of CNNM using some examples.

We first illustrate the advantage of "Symmetric dissimilarity". In general, real-world human image data of the limited sample size suffer from large intra-class variations and small inter-class differences, which can be simplified in Fig. 4. Let $d$ be the query sample. $a$ and $e$ are assigned as the corpus samples. $d$ and $a$ are in the same class, but $e$ is from the different class. By Euclidean distance, $e$ will rank before $a$ with regard to $d$.

Next, we consider Rank-Order distance and "Symmetric dissimilarity". Each sample ranks itself first, so rank 


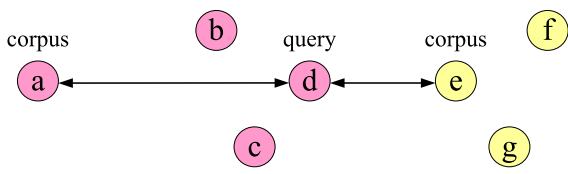

Fig. 4 An example of comparison between "Symmetric dissimilarity" and Rank-Order distance. Samples' classes can be distinguished by colors.

order lists for $a, d$, and $e$ are abcdegfh, dcbegfah, and egfdcbha, respectively, with integer rank orders from 0 to 7. Zhu et al.'s Rank-Order distance fails because of the large intra-class variations and small inter-class differences: $D(a, d)=[(6+2+1+0)+(3+2+1+4+5+6+0)] / 3=10$ and $D(d, e)=[(3+4+5+0)+(3+4+5+0)] / 3=8$. However, our "Symmetric dissimilarity" succeeds by exploiting the common-near-neighbor information with a moderate "Fixed-number" $n=2: D(a, d)=(6+2)+(3+2)=13$ and $D(d, e)=(3+4)+(3+4)=14$.

Besides this concrete example, we now provide a statistical illustration for CNNM. Synthetic data can help us further elaborate our proposal [19]. We randomly generate three separate, Gaussian-distributed datasets for use as class samples (for a class size of 40). For fairness, corpus and query points are assigned for ten times by randomly halving the data. We connect each query point to its top-ranked corpus point measured by CNNM, with $n$ set to half of the average sample number in each class and $\lambda$ tentatively set to 1 and 0 , respectively. We then compare CNNM (for which $n=20$ and $\lambda=1$ ) to the baseline dissimilarity measured by Euclidean distance and to Zhu et al.'s RankOrder distance $D^{\text {Rank-Order }}$ given by Eq. (1). We also compare our "Fixed-number" based "Symmetric dissimilarity" (for which $n=20$ and $\lambda=0$ ) to Zhu et al.'s flexible-number based $D_{a}(b)+D_{b}(a)$ in $D^{\text {Rank-Order }}$. All the experiments use the same generated data and the same corpus-query splits.

Note that, our synthetic data based experiments carried out here are essentially different from Zhu et al.'s, though they seem to be similar. Zhu et al. used experiments to demonstrate the robustness of Rank-Order distance during clustering. For clusters with varying density/shape/size, they plotted all the edges between points whose Rank-Order distances are smaller than a given threshold [17]. We use experiments to visualize the advantage of CNNM evaluated by recognition/rankings. These experiments are relevant to the re-identification issue, but differ from those in Zhu et al.'s work to demonstrate the clustering effect.

The lines shown in Fig. 5 display accumulative results for ten-fold cross-validation. From these, we can confirm that CNNM yields the least false lines among all the compared methods, and that even our common-near-neighbor informatic "Symmetric dissimilarity" wins both $D_{a}(b)+D_{b}(a)$ and $D^{\text {Rank-Order }}$ of Zhu et al.'s as well.

The Gaussian-distributed synthetic data dismissed in Fig. 5 is used to illustrate the advantage of CNNM. Realworld human image data are high-dimensional, and it is impossible to visualize these data directly. Synthetic low-

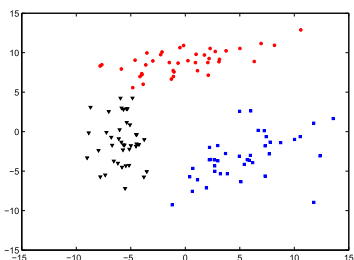

(a) Synthetic data

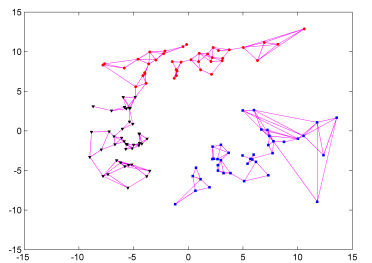

(c) Rank-Order distance (Zhu et al.'s)

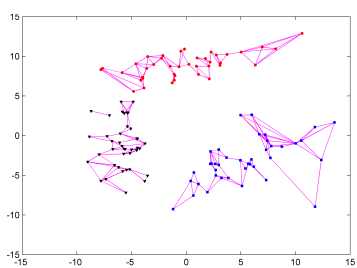

(e) Symmetric dissimilarity (Ours)

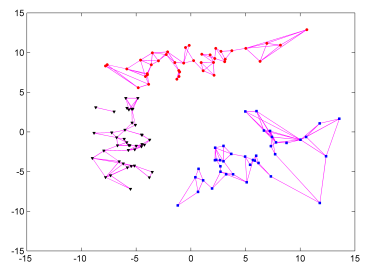

(b) CNNM (Ours)

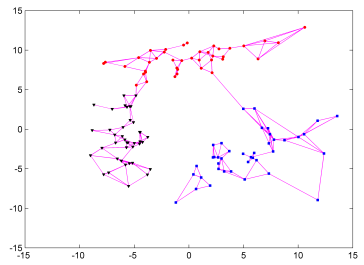

(d) Euclidean distance

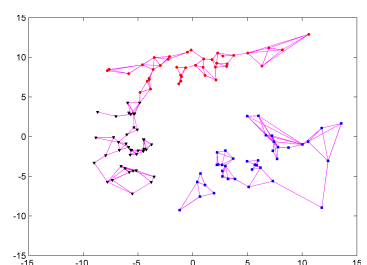

Fig. 5 Synthetic data is generated randomly to test the performance of CNNM and "Symmetric dissimilarity". Classes are labeled by distinct colors. A magenta line is used to connect each query point with its top-ranked corpus point.

dimensional data simulations can be useful to some extent, and there seems to be no better alternative than this approach. Honestly, these simple synthetic data cannot directly represent the complex real distribution of the data in the actual re-identification application. For these synthetic data, most samples in the same class reside closer to each other than those from different classes. Though this is an expected property required for re-identification, the real data distribution rarely satisfies this property. Hence, for those real data, we need to use metric learning to improve the intra-class compactness with regard to the inter-class separation before performing CNNM. Though being not perfect perhaps, such improvement to a certain degree makes the real data distribution satisfy that property as far as possible, and thus similar to the situation of the synthetic data distribution. From this perspective, the synthetic data visualization can indirectly represent the real distribution of the data. Furthermore, in Sect. 3, experiments across several widely-used benchmark datasets will have demonstrated the effectiveness of CNNM in a learned metric space using real data. These synthetic data based experiments opportunely complement them. Overall, synthetic data and real data contribute to a more convincing demonstration on the advantage of our proposed method.

\subsection{Metric Space Construction with OMRR}

CNNM is based on the direct distance measure on the low level, so the performance of CNNM is more or less influ- 
enced by the underlying distances. To guarantee the effectiveness of CNNM, it is indispensable to construct and improve a good metric space therefore. In a noisy feature space, the intruders and outliers of each class may degrade the performance of CNNM. To avoid this, we need to denoise the rank order lists for CNNM measurement by improving intra-class compactness relative to inter-class separation. Coincidentally, OMRR can benefit CNNM by optimizing list-wise rankings.

Given queries set $Q=\left\{q \mid q \in \mathbb{R}^{d}\right\}$ and corpus set $\mathcal{X}=\left\{x_{q i} \mid x_{q i} \in \mathbb{R}^{d}\right\}$, suppose $\phi_{q i}\left(x_{q i}, q\right)$ is used to denote a kind of relative feature representation of a corpus sample $x_{q i}$ w.r.t. $q$ and suppose $w$ is the metric we intend to optimize. A desired ranking model could be $g_{w}\left(x_{q i}\right)=w^{T} \phi_{q i}\left(x_{q i}, q\right)$, which scores each $x_{q i}$ and the ranking is done by sorting the score in a descending order. Let $y \in \mathcal{Y}$ is a ranking of $\mathcal{X}$ w.r.t the query $q$, and $\psi(q, y, \mathcal{X}) \in \mathbb{R}^{d}$ be a vector-valued joint feature map as defined in [15]. Then, optimizing $w$ for the ranking model $g_{w}\left(x_{q i}\right)$ is equivalent to optimizing the following model based on $\psi(q, y, \mathcal{X})$ :

$$
\arg \min _{w} \frac{1}{2}\|w\|^{2}+\frac{C}{|Q|} \sum_{q} \xi_{q}
$$

s.t.

$$
\begin{gathered}
w^{T} \psi\left(q, y_{q}^{*}, \mathcal{X}\right) \geq w^{T} \psi(q, y, \mathcal{X})+\Delta\left(y_{q}^{*}, y\right)-\xi_{q}, \\
\forall q, y \neq y_{q}^{*} ; \\
\xi_{q} \geq 0, \forall q,
\end{gathered}
$$

where $y_{q}^{*}$ is the ground truth ranking of $\mathcal{X}$ for a given $q \in Q$, $\xi_{q}$ is the slack variable, $C$ is the trade-off parameter, and $\Delta\left(y_{q}^{*}, y\right)$ is the loss function to penalize predicting $y$ instead of $y_{q}^{*}$, defined by $\Delta\left(y_{q}^{*}, y\right)=1-S_{\mathrm{MRR}}(q, y)$, in which

$$
S_{\mathrm{MRR}}(q, y)=\frac{1}{|Q|} \sum_{q \in Q}\left\{\begin{aligned}
1 / r_{q}, & r_{q}<k ; \\
0, & r_{q} \geq k,
\end{aligned}\right.
$$

where $k$ is a threshold value that can be assigned a priori, and $r_{q}$ is the rank order of ground-truth corpus sample with regard to the query $q$.

Conceptually, the reciprocal rank of a query response is the multiplicative inverse of the rank of the first correct match, and MRR is the average of such reciprocal ranks of results over the whole query set. To a degree, this coincides with practical performance expectations. Since only the rank of the first correct match is taken into account, the ranks of both other correct matches and any incorrect matches are arbitrary. Thus, there will be multiple ranking instantiations for a given ground truth. In practice, MRR is quite reasonable, and there seems to be no significantly better options [15], [20].

Given a large number of constraints, it is reasonable to approximate a solution. For this purpose, the cutting-plane algorithm fits nicely. The idea of a cutting-plane algorithm is to approximate either the constraint set or the epigraph of the cost function by intersecting a limited number of half spaces. It usually refines the approximation incrementally,

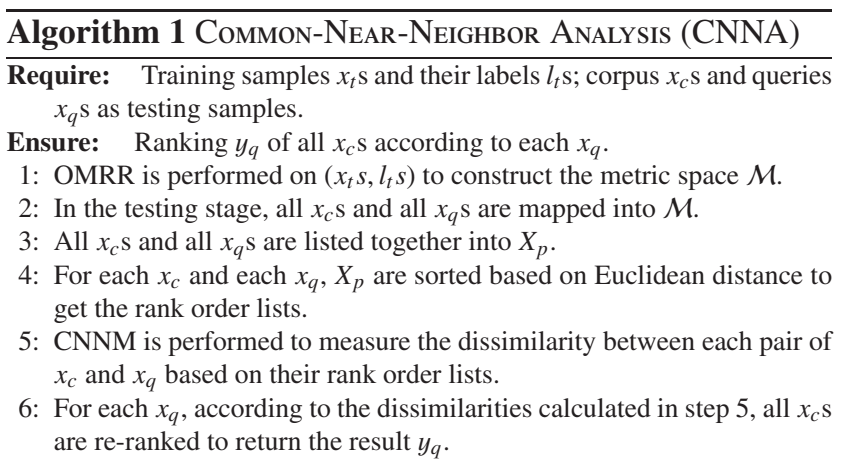

by generating additional half spaces through the use of subgradients [21], [22].

After learning, a new metric space can be constructed. In this new space, intra-class distances should be smaller than inter-class distances, thus helping to improve the performance of CNNM.

CNNM is a high-level dissimilarity measure that is based on the rank order lists formed by the low-level dissimilarity measure. Hence, the more reliable low-level dissimilarity measure is, the better performance CNNM will have. To enhance the quality of rank order lists, we employ OMRR to improve the intra-class compactness with regard to the inter-class separation before measuring the low-level dissimilarity among samples. From a pre-processing standpoint, OMRR plays a de-noising role for these human image data. If the original data are sufficiently separable, even without OMRR, CNNM can work effectively. Accordingly, there is no direct relationship between the performance of the distance metric learning and the choice of the "Fixednumber" $n$, to be frank.

The overall algorithm of CNNA is presented in Algorithm 1.

\section{Experiments and Results}

\subsection{Dataset Description and Experimental Setup}

We tested our CNNA method on public available datasets VIPeR [23], ETHZ [24], and i-LIDS [25] (of which representative samples are shown in Fig. 6) and averaged the results for ten executions against random training-testing data splits. For fairness, we used the same samples from the assigned dataset for comparison. Experiments were conducted in two groups: parameter discussion and method demonstration. In parameter discussion, we sought to confirm the suitable settings of tunable parameters for CNNM, including the "Fixed-number" $n$ and trade-off parameter $\lambda$. In method demonstration, we sought to confirm the importance of metric space selection and the superiority of our modeling to Zhu et al.'s Rank-Order distance, since both are components of CNNA. We also evaluated the effectiveness of our CNNA method in comparison to related state-of-the-art methods.

The VIPeR dataset consists of 632 image pairs corresponding to 632 unique pedestrians. Each pedestrian image 


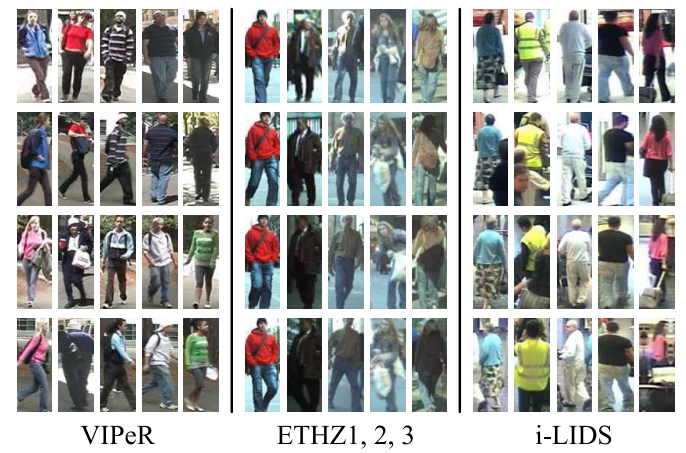

Fig. 6 Exemplars from dataset VIPeR, ETHZ1,2,3, and i-LIDS, showing the real-world challenge of re-identification.

pair has been taken from arbitrary viewpoints under varying illumination conditions, making VIPeR one of the most challenging datasets for person re-identification [23]. The ETHZ datasets are composed of three video sequences of crowded street scenes captured by two moving cameras. Of these, we utilized three subsets extracted by Schwartz and Davis for person re-identification testing [26]. These have smaller pose and viewpoint variations, but more illumination changes, than the VIPeR dataset. There are 83 pedestrians within 4857 images in SEQ1 ("ETHZ1"), 35 pedestrians within 1936 images in SEQ2 ("ETHZ2"), and 28 pedestrians within 1762 images in SEQ3 ("ETHZ3"). The i-LIDS MCTS dataset is a publicly available video dataset captured at a busy airport arrival hall using a multi-camera CCTV network, and thus constitutes a true real-world dataset. From these videos, a subset of 479 images of 119 pedestrians, denoted by i-LIDS, was extracted by Zheng et al. for testing their context-based pedestrian re-identification method [25]. For each identity, there are 2 to 8 images taken from nonoverlapping cameras. This i-LIDS dataset suffers from more severe pose variations and occlusion than VIPeR and ETHZ1,2,3. For all of the above datasets, we normalized images to $48 \times 128$ pixels, the same size as images in VIPeR.

Because feature representation is not the focus of this paper, we did not tune it for a better performance. Considering the complexity of the issue and following the state-ofart, CNNA uses a signature concatenation of two widelyused features: Weighted HSV Color Histogram (WHSV) and Dense Sampled Color Histogram (DSCH), denoted by WHSV+DSCH. WHSV can handle changes in viewpoint and pose by considering both global color information and structural properties of the human body [4], [5]. DSCH can tackle changes in illumination and occlusion in terms of its cell-based statistical local color description and a global dense sampling of these cells [9]. Since both WHSV and DSCH have their own strengths and exploit human appearance information from different perspectives, they make a powerful combination.

It is worth mentioning that, we selected WHSV to build the signature because this feature can not only provides a good performance, but also ensure a fair comparison with state-of-the-art methods. To form the WHSV feature rep- resentation, we obtained the silhouette masks based on the same STEL (STructure ELement) model as in [4], [5]. A silhouette mask containing only foreground pixel values was acquired for each person by inferring over the STEL generative model [27]. This model captures the general structure of an image class as a blend of several component segmentations, isolating meaningful parts that exhibit tight distributions over the image measurements. This model was customized for foreground/background separation by setting two components and two parts corresponding to the foreground and background, and learned beforehand using a subset of VIPeR not for testing. In fact, the authors of WHSV have mentioned that, to segment out the pedestrian from the background for WHSV, it is not important which dataset is used to learn the STEL model. Additionally, there is one convenient way to acquire the WHSV feature representation and silhouette masks for several widely-used benchmark datasets. They are available on line provided by Loris Bazzani ${ }^{\dagger}$.

We randomly halved the persons of each dataset for training-testing use. First, the training data was used to construct a metric space for the testing data. Then, for each person, one image was randomly selected to form the corpus, leaving the remaining images for the query. We selected each image from the query side and matched it to each image from the corpus side according to dissimilarity. While calculating the dissimilarity between the query image and corpus image by CNNM, we used those remaining unlabeled query and corpus images to help form the rank order lists. We then obtained the correct match upon these dissimilarities. The entire procedure was repeated for 10 times, and the average results were plotted as CMC (Cumulative Match Characteristic) curves, the most widely used evaluation criterion for person re-identification. CMC illustrates how the performance (recognition/re-acquisition rate) improves as the number of requested images increases.

\subsection{Parameter Discussion}

\subsubsection{Fixed-Number}

To discuss how important the "Fixed-number" $n$ is for "Symmetric dissimilarity" of CNNM in the OMRR metric space, we sampled ETHZ datasets randomly to produce five hand-crafted datasets with the same number of images per person: 2, 4, 8, 16, and 32 (denoted by ETHZ_N2, ETHZ_N4, ETHZ_N8, ETHZ_N16, ETHZ_N32, respectively). We also included another hand-crafted dataset with 2 images per person from i-LIDS (denoted by i-LIDS_N2). Combining these with the VIPeR dataset (for which the number of images per person is always 2), we evaluated the possible relationship between the most suitable "Fixednumber" and the number of images per person, with respect to the performance of "Symmetric dissimilarity". Our results are described by the condensed measure MRR shown

\footnotetext{
†http://www.lorisbazzani.info/code-datasets/sdalf-descriptor/
} 


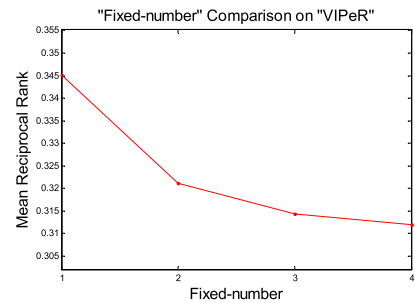

(a)

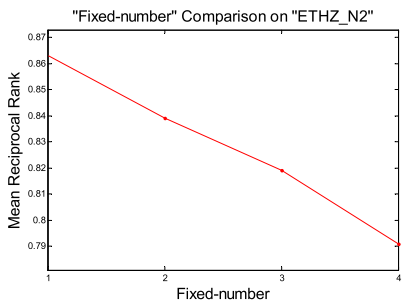

(b)

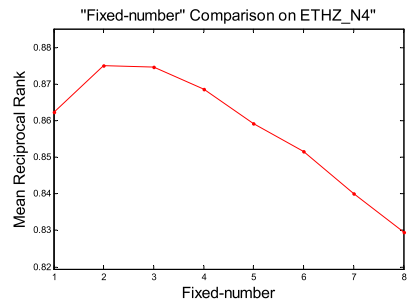

(c)

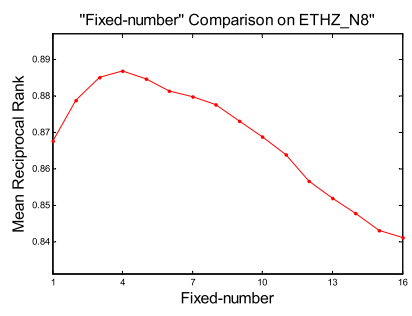

(d)

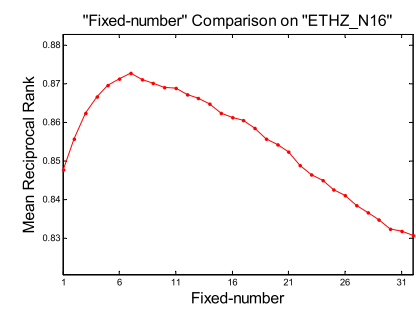

(e)

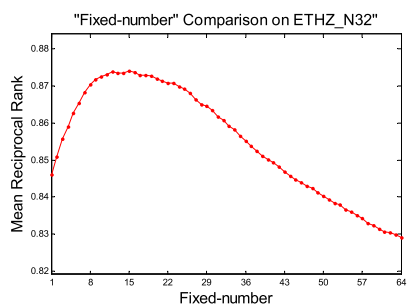

(f)

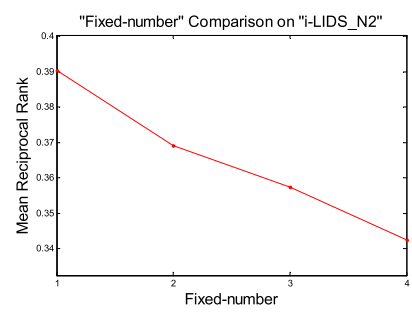

(g)

Fig. 7 Performance of "Symmetric dissimilarity" for different "Fixed-number" recommendation in terms of MRR.

in Fig. 7. MRR was suggested and detailed in [15], and is given by MRR $=\frac{1}{|Q|} \sum_{q \in Q} \frac{1}{r_{q}}$, where $q$ is a query belong to a queries set $Q$, and $r_{q}$ is the rank of the first correct match as the query response. MRR offers an overall evaluation of the rankings. From the compared results, we can see that, coincident with our recommendation, when the "Fixed-number" is set to approximately half the number of images per person, "Symmetric dissimilarity" performs best.

As expected, the "Fixed-number" $n$ is related to the sample number of each person rather than the number of the people in the corpus. A suitable neighborhood size is required to exploit the common-near-neighbor information for differentiating samples' classes, considering that samples from the same class probably share more similar neighborhood structure than those from different classes. To model a discriminative "Symmetric dissimilarity", we suggest the use of the "Fixed-number" $n$ to cover the neighboring samples from the same class as far as possible to maximize the capture of the within-class sample distribution information for each sample. Thus, it is necessary to concern the relationship between the "Fixed-number" $n$ and the sample number of each person. Further, though the rank order lists contain the people in the corpus, there is no indicator of a relationship between the "Fixed-number" $n$ and the number of the people in the corpus for producing a most discriminative "Symmetric dissimilarity". This claim has been demonstrated by the experimental results on the datasets of different class sizes and class numbers in Fig. 7, from which, we can see the relationship between the "Fixed-number" $n$ and the sample number of each person rather than the number of the people in the corpus.

\subsubsection{Trade-Off Parameter}

Trade-off parameter $\lambda$ plays a role in balancing "Symmetric dissimilarity" and "Asymmetric dissimilarity" for CNNM
Table 1 Performance of CNNM for different trade-off parameter settings and different numbers of images per person in terms of MRR.

\begin{tabular}{|c|c|c|c|c|c|}
\hline$\lambda$ & VIPeR & ETHZ_N2 & ETHZ_N8 & ETHZ_N16 & i-LIDS_N2 \\
\hline 0.001 & 0.3453 & 0.8640 & 0.8870 & 0.8711 & 0.4701 \\
0.01 & 0.3453 & 0.8640 & 0.8872 & $\mathbf{0 . 8 7 1 2}$ & 0.4701 \\
0.1 & 0.3458 & 0.8642 & $\mathbf{0 . 8 8 7 5}$ & 0.8705 & 0.4703 \\
1 & $\mathbf{0 . 3 4 8 6}$ & $\mathbf{0 . 8 6 8 6}$ & 0.8847 & 0.8675 & $\mathbf{0 . 4 7 7 3}$ \\
10 & 0.3390 & 0.8673 & 0.8695 & 0.8532 & 0.4706 \\
100 & 0.3348 & 0.8638 & 0.8604 & 0.8436 & 0.4698 \\
1000 & 0.3346 & 0.8638 & 0.8601 & 0.8424 & 0.4698 \\
\hline
\end{tabular}

in the OMRR metric space. We conducted experiments on VIPeR, ETHZ_N2, ETHZ_N8, ETHZ_N16, and i-LIDS_N2 using the recommended "Fixed-number" setting to discuss how the number of images per person influences "Symmetric dissimilarity" and "Asymmetric dissimilarity", and possibly to clarify the characteristics of "Symmetric dissimilarity" and "Asymmetric dissimilarity" for different class sizes. Our results are described by MRR in Table 1. Note that when the number of images per person is small, there is not enough common-near-neighbor information to differentiate the contributions of "Symmetric dissimilarity" and "Asymmetric dissimilarity" to the overall performance of CNNM. As the number of images per person increases, the superior competence of "Symmetric dissimilarity" to "Asymmetric dissimilarity" becomes clearer. From this result, we conclude that the number of images per person should directly impact the selection of $\lambda$ in CNNM, favoring "Symmetric dissimilarity" for higher numbers of images.

We justified this assertion using the original VIPeR, ETHZ1, ETHZ2, ETHZ3, and i-LIDS datasets, which have approximately averages of $2,60,40,60$, and 4 images per person, respectively. Experimental results are described by MRR in Table 2, where $\lambda=\infty$ means only "Asymmetric dissimilarity" works. Note that CNNM in the OMRR metric space performs best on VIPeR when $\lambda=1$, on ETHZ1,2,3 when $\lambda=0$, and on i-LIDS when $\lambda=0.1$. Indeed, re- 
Table 2 Performance of CNNM against different benchmark datasets for different trade-off parameter settings in terms of MRR.

\begin{tabular}{|c|c|c|c|c|c|}
\hline$\lambda$ & VIPeR & ETHZ1 & ETHZ2 & ETHZ3 & i-LIDS \\
\hline 0 & 0.3452 & $\mathbf{0 . 8 6 0 6}$ & $\mathbf{0 . 8 9 0 4}$ & $\mathbf{0 . 9 7 7 3}$ & 0.5155 \\
0.001 & 0.3453 & 0.8606 & 0.8904 & 0.9773 & 0.5157 \\
0.01 & 0.3453 & 0.8605 & 0.8903 & 0.9772 & 0.5160 \\
0.1 & 0.3458 & 0.8597 & 0.8897 & 0.9764 & $\mathbf{0 . 5 1 7 1}$ \\
1 & $\mathbf{0 . 3 4 8 6}$ & 0.8498 & 0.8817 & 0.9657 & 0.5168 \\
10 & 0.3390 & 0.8233 & 0.8589 & 0.9330 & 0.4978 \\
100 & 0.3348 & 0.8074 & 0.8406 & 0.9205 & 0.4880 \\
1000 & 0.3346 & 0.8052 & 0.8394 & 0.9194 & 0.4968 \\
$\infty$ & 0.3276 & 0.8045 & 0.8385 & 0.9183 & 0.4822 \\
\hline
\end{tabular}

sults suggest that the number of images per person is the only factor that significantly influences the performance of "Symmetric dissimilarity". As is well known, VIPeR has an extremely small sample size but a very complicated data distribution, such that when " $n=1$ ", there are no near neighbors available in "Symmetric dissimilarity", weakening it relative to "Asymmetric dissimilarity".

CNNM works best when "Symmetric dissimilarity" does not need to collaborate with "Asymmetric dissimilarity" in the cases of datasets ETHZ1, ETHZ2, and ETHZ3 $(\lambda=0)$. This validates the performance of common-nearneighbor information measurement using "Symmetric dissimilarity" for cases in which the number of images per person is large enough. CNNM shows its advantages clearly as well when processing the i-LIDS dataset, with its small number of diverse images per person. By using "Asymmetric dissimilarity" to tackle the asymmetric ranking problem, CNNM can still perform robustly against this challenging dataset.

To sum up, it is not exact that the smaller $\lambda$ is, the better performance of CNNM tends to achieve. The recommended trade-off parameter tends to be influenced by the class size. When the class samples are sufficient, there will be enough available resources for the "Symmetric dissimilarity" to perform well. Then, the within-class distribution information can help to offset the deviation problem of the isolated samples to a certain degree, and thus provide the robustness to the asymmetric ranking problem, so the "Asymmetric dissimilarity" weights small usually. For the very small class size case, the "Symmetric dissimilarity" loses some capability, and the "Asymmetric dissimilarity" can compensate for it for a robust performance, so the "Asymmetric dissimilarity" weights larger than usual.

\subsection{Method Demonstration}

\subsubsection{Metric Space Selection}

To verify the suitability of metric space selection, we tested CNNM in different comparable metric spaces, including Euclidean metric space, SDALF metric space, LMNN metric space, and OMRR metric space. Combining WHSV and $\mathrm{DSCH}$ has been proved more effective than original SDALF [15], so the SDALF metric space is here generalized to indicate measuring distance between suitable feature representations with the Bhattacharyya metric.

From the results shown in Fig. 8 (a), we can see that, for CNNM, the metric space constructed by OMRR significantly outperforms other metric spaces on different datasets. Further, note that, in a metric space learned by LMNN, which aims at optimizing a metric space for classification rather than list-wise ranking, CNNM does not work well. This clearly verifies the importance of metric space selection.

The experiments on changing the metric space for CNNA with results presented in Fig. 8 (a) show that CNNA is a flexible model which may use any metric space for CNNM. OMRR is so far the best we've found and we explained why it is better than the others for assisting CNNM. There may be better ones showing up in the future. It is totally fine to try the new ones with CNNA, so we plan to not emphasize too much on a specific metric learning model like OMRR and avoid pure discussion about metric learning itself. OMRR does provide a good metric space for CNNM, but it is also observed that CNNM brings significant performance improvements to various metric learning models including OMRR.

\subsubsection{Modeling Validation}

To assess and validate the superiority of CNNM, we compared it with Zhu et al.'s Rank-Order distance modeling in their Euclidean space. For fairness, we used the same feature representation WHSV+DSCH for CNNM and Zhu et al.'s modeling, but also tried moving their modeling into our OMRR metric space. From the results shown in Fig. 8 (b), we see that, for person re-identification, our modeling is more effective than Zhu et al.'s even in their Euclidean metric space. Also note that, as expected, a metric space learned by optimizing list-wise ranking provides a platform for better performance for Zhu et al.'s Rank-Order distance.

\subsubsection{Method Evaluation}

We also compared our CNNA method to related state-ofthe-art methods [4], [5], LMNN [10], OMRR [15], KISSME [13], and RDC [11], using our recommended parameter-settings (for VIPeR, $\lambda=1, n=1$; for ETHZ1, $\lambda=0, n=30$; for ETHZ2, $\lambda=0, n=20$; for ETHZ3, $\lambda=0, n=30$; for i-LIDS, $\lambda=0.1, n=2$ ). From the results in Fig. 8 (c), it can be seen that CNNA always remarkably outperforms the runner-ups, while the performances of its competitors fluctuate across different datasets.

Each dataset has its distinct difficulties to be addressed, though other challenges may also present, as exemplified in Fig. 6. From the experimental results, we can see CNNA is more robust than other methods to viewpoint variation (VIPeR), illumination variation (ETHZ1,2,3), pose variation (i-LIDS), and occlusion (i-LIDS). This validates the competency of CNNA for the challenging real-world human image data.

The rank order lists of CNNA are formed by images 

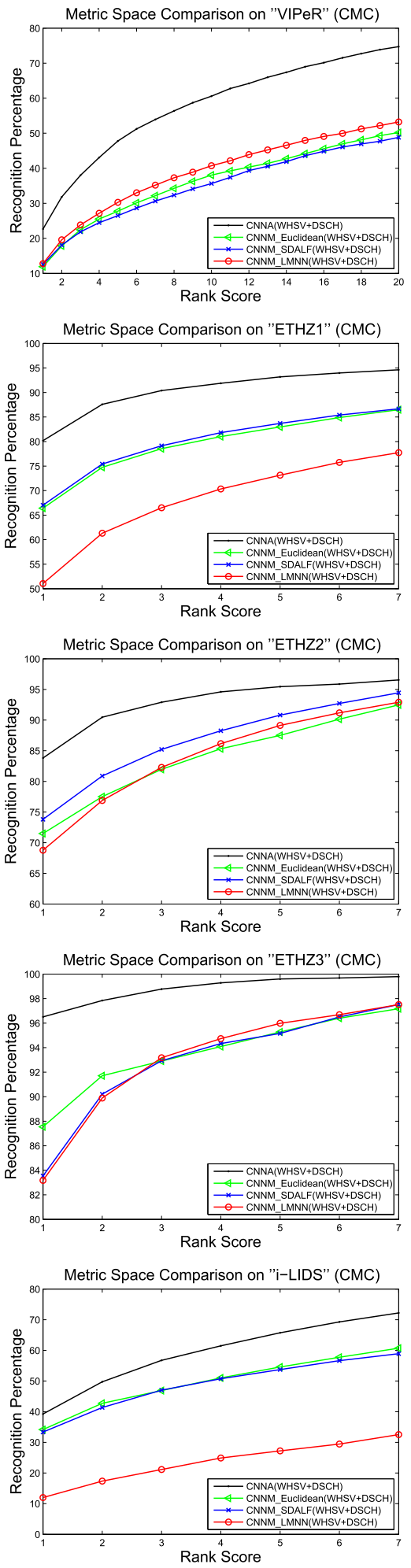

(a) Metric Space Comparison
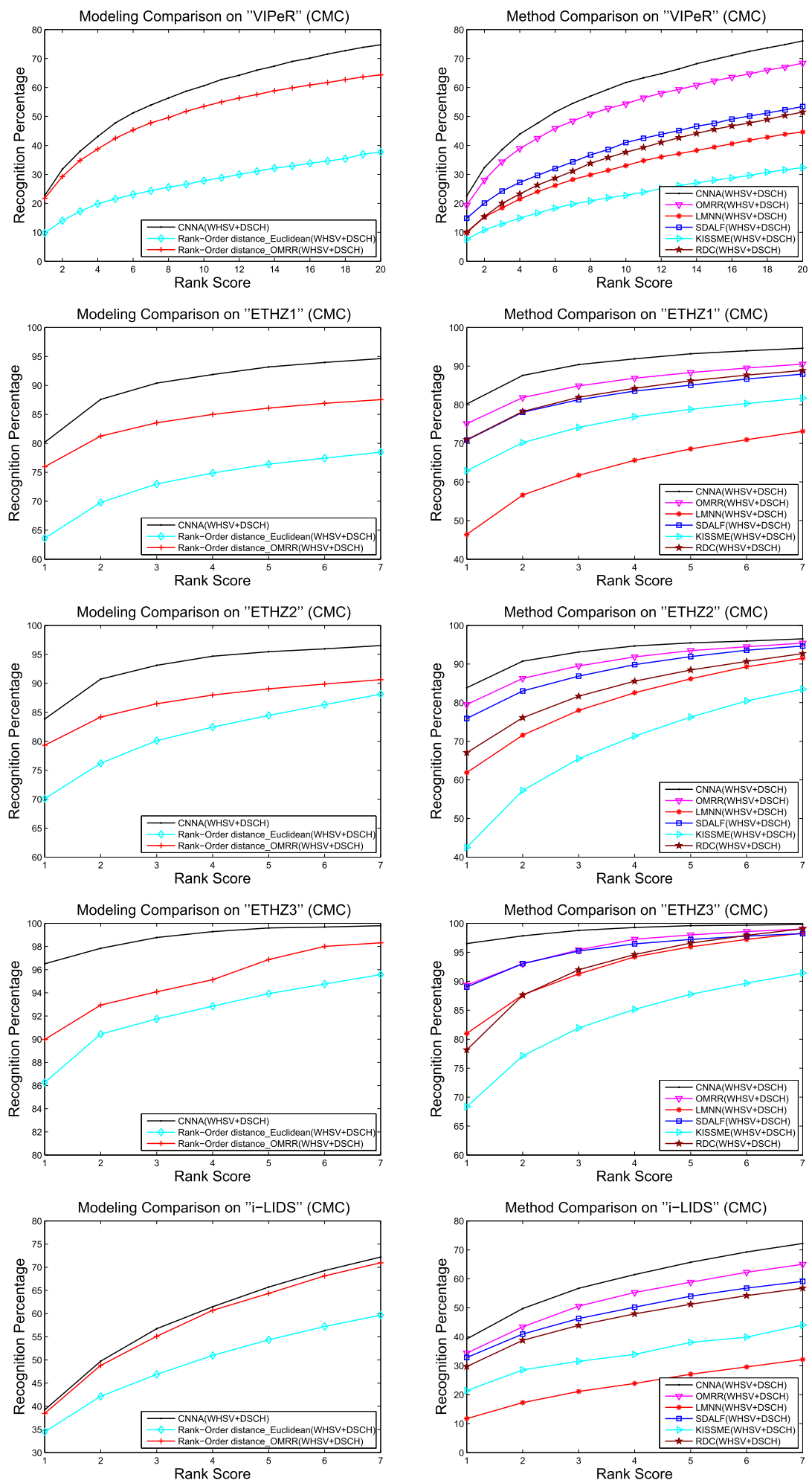

(b) Modeling Comparison

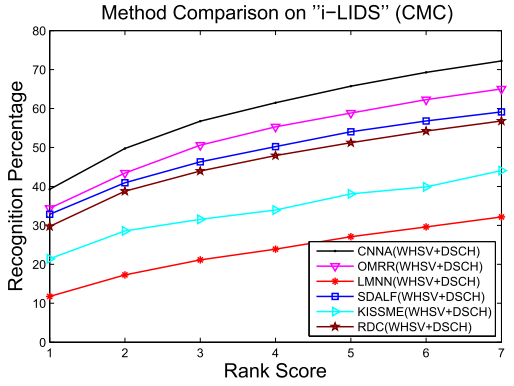

(c) Method Comparison

Fig. 8 Metric space, modeling, and method comparison for VIPeR, ETHZ1,2,3, and i-LIDS.

from the query side and corpus side. When each class contains multiple images, CNNA can perform effectively with a suitable choice of the "Fixed-number" $n$, as demonstrated on ETHZ1,2,3 and i-LIDS. However, VIPeR represents a single-shot vs. single-shot re-identification problem, i.e., for each identity, there is one image on the query side and corpus side in ground-truth during the re-identification procedure. In this case, there are merely 2 images in each class. 
Even so, we can still see the strong compatibility of CNNA towards this extremely small sample size situation, with the help of the side information.

Honestly, the single-shot vs. single-shot case is one of the most specific and challenging problems for reidentification. The extremely small sample size limits the power of metric learning, so, in a learned metric space, the query sample usually unsatisfactorily resides far from the corpus sample of the same class, but near to those from different classes [20]. If we select a larger neighborhood size for Eq. (3), samples from different classes will be easily included. This situation is adverse for characterizing the discriminatory within-class sample distribution for each sample, thereby having a negative impact on "Symmetric dissimilarity". Similarly, the use of a flexible neighborhood is also prone to incur too many intruders, and thus degrades the discriminative ability of Zhu et al.'s Rank-Order distance.

Based on the analytical and experimental discussions for the "Fixed-number", we adopted $n=1$. Inviting no additional neighbors is better than taking the risk of introducing intruders. When $n=1$, the asymmetric ranking problem is obtrusive. This problem has been suggested to be tackled by collaborating "Symmetric dissimilarity" and "Asymmetric dissimilarity" in our model. Therefore, in this case, in the learned metric space, besides the advantage of inherent rank order quantization, the use of a suitable "Fixed-number" and the collaborative model still support the superiority of our proposal.

\section{Conclusion}

This paper has proposed a novel method CNNA for person re-identification. It exploits a new dissimilarity modeling approach by analyzing common-near-neighbor information in a suitably constructed metric space. Its theoretical advantages are borne out by experiments on diverse datasets. In future work, we would like to develop and apply CNNA to real-world problems in human tracking.

\section{Acknowledgments}

This work was supported by "R\&D Program for Implementation of Anti-Crime and Anti-Terrorism Technologies for a Safe and Secure Society", Funds for integrated promotion of social system reform and research and development of the Ministry of Education, Culture, Sports, Science and Technology, the Japanese Government.

\section{References}

[1] D. Gianfranco, T. Sebastian, P. Tu, and J. Rittscher, "Appearancebased person reidentification in camera networks: Problem overview and current approaches," J. Ambient Intelligence and Humanized Computing, vol.2, pp.127-151, 2011.

[2] S. Bak, E. Corvee, F. Bremond, and M. Thonnat, "Person reidentification using haar-based and dcd-based signature," Proc. 7th International Conference on Advanced Video and Signal Based Surveillance, AVSS, pp.1-8, Aug. 2010.
[3] B. Loris, C. Marco, P. Alessandro, F. Michela, and M. Vittorio, "Multiple-shot person re-identification by he signature," Proc. 20th International Conference on Pattern Recognition, ICPR, pp.14131416, Istanbul, Turkey, Aug. 2010.

[4] M. Farenzena, L. Bazzani, A. Perina, V. Murino, and M. Cristani, "Person re-identification by symmetry-driven accumulation of local features," Proc. 23rd Conference on Computer Vision and Pattern Recognition, CVPR, pp.2360-2367, San Francisco, USA, June 2010.

[5] L. Bazzani, M. Cristani, and V. Murino, "Symmetry-driven accumulation of local features for human characterization and reidentification," Comput. Vis. Image Understand., vol.117, pp.130144, 2013.

[6] B. Slawomir, C. Etienne, B. Francois, and T. Monique, "Multipleshot human re-identification by mean riemannian covariance grid," Proc. 8th International Conference on Advanced Video and SignalBased Surveillance, AVSS, pp.179-184, Klagenfurt, Austria, Aug. 2011.

[7] R. Zhao, W. Ouyang, and X. Wang, "Unsupervised salience learning for person re-identification," Proc. 26th Conference on Computer Vision and Pattern Recognition, CVPR, pp.3586-3593, Portland, Oregon, USA, June 2013.

[8] Y. Liu and J. Rong, "Distance metric learning: A comprehensive survey," tech. rep., Department of Computer Science and Engineering, Michigan State University, 2006.

[9] M. Dikmen, E. Akbas, T.S. Huang, and N. Ahuja, "Pedestrian recognition with a learned metric," Proc. 10th Asian Conference on Computer Vision, ACCV, pp.501-512, New Zealand, UK, Nov. 2010.

[10] K.Q. Weinberger and L.K. Saul, "Distance metric learning for large margin nearest neighbor classification," J. Machine Learning Research, vol.10, pp.207-244, Feb. 2009.

[11] W. Zheng, S. Gong, and T. Xiang, "Re-identification by relative distance comparison," IEEE Trans. Pattern Anal. Mach. Intell., vol.35, pp.653-668, March 2013.

[12] B. Prosser, W. Zheng, S. Gong, and T. Xiang, "Person reidentification by support vector ranking," Proc. British Machine Vision Conference, pp.21.1-21.11, Wales, UK, Aug. 2010.

[13] M. Koestinger, M. Hirzer, P. Wohlhart, P.M. Roth, and H. Bischo, "Large scale metric learning from equivalence constraints," Proc. 25th Conference on Computer Vision and Pattern Recognition, CVPR, pp.2288-2295, Providence, Rhode Island, June 2012.

[14] W. Li and X. Wang, "Locally aligned feature transforms across views," Proc. 26th Conference on Computer Vision and Pattern Recognition, CVPR, pp.3594-3601, Portland, Oregon, USA, June 2013.

[15] Y. Wu, M. Mukunoki, T. Funatomi, and M. Minoh, "Optimizing mean reciprocal rank for person re-identification," Proc. 8th International Conference on Advanced Video and Signal-Based Surveillance, AVSS, pp.408-413, Klagenfurt, Austria, Aug. 2011.

[16] B. McFee and G. Lanckriet, "Metric learning to rank," Proc. 27th International Conference on Machine Learning, ICML, pp.775-782, Haifa, Israel, June 2010.

[17] C. Zhu, F. Wen, and J. Sun, "A rank-order distance based clustering algorithm for face tagging," Proc. 24th Conference on Computer Vision and Pattern Recognition, CVPR, pp.481-488, Colorado Springs, USA, June 2011.

[18] W. Li, Y. Wu, M. Mukunoki, and M. Minoh, "Common-nearneighbor analsis for person re-identification," Proc. 19th International Conference on Image Processing, ICIP, pp.1621-1624, Florida, USA, Sept. 2012.

[19] W. Li, Y. Wu, M. Mukunoki, and M. Minoh, "Bi-level relative information analysis for multiple-shot person re-identification," IEICE Trans. Inf. \& Syst., vol.E96-D, no.11, pp.2450-2461, Nov. 2013.

[20] W. Li, Y. Wu, M. Mukunoki, and M. Minoh, "Coupled metric learning for single-shot versus single-shot person re-identification," Optical Engineering, pp.027203-1-10, Feb. 2013.

[21] T. Joachims, T. Finley, and C.N.J. Yu, "Cutting-plane training of 
structural svms," Machine Learning, vol.77, pp.27-59, 2009.

[22] B. Stephen and V. Lieven, Convex Optimization, Cambridge University Press, March 2004.

[23] D. Gray and H. Tao, "Evaluating appearance models for recognition, reacquisition, and tracking," Proc. 10th International Workshop on Performance Evaluation for Tracking and Surveillance, PETS, pp.41-47, Rio de Janeiro, Brazil, Oct. 2007.

[24] A. Ess, B. Leibe, and L.V. Gool, "Depth and appearance for mobile scene analysis," Proc. 11th International Conference on Computer Vision, ICCV, pp.1-8, Rio de Janeiro, Brazil, Oct. 2007.

[25] W.S. Zheng, S.G. Gong, and T. Xiang, "Associating groups of people," Proc. 20th British Machine Vision Conference, BMVC, pp.23.1-23.11, London, UK, Sept. 2009.

[26] W.R. Schwartz and L.S. Davis, "Learning discriminative appearance-based models using partial least squares," Proc. XXII Brazilian Symposium on Computer Graphics and Image Processing, SIBGRAPI, pp.322-329, Rio de Janeiro, Brazil, Oct. 2009.

[27] N. Jojic, A. Perina, M. Cristani, V. Murino, and B. Frey4, "Stel component analysis: Modeling spatial correlations in image class structure," Proc. 22th Conference on Computer Vision and Pattern Recognition, CVPR, pp.2044-2051, Los Alamitos, CA, USA, June 2009.

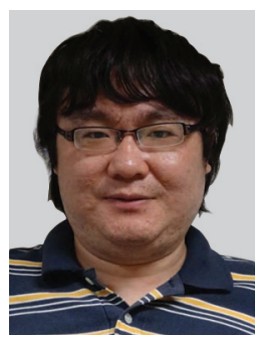

Wei $\mathbf{L i} \quad$ is currently a specially appointed researcher of the Institute of Scientific and Industrial Research at Osaka University. He received the bachelor's degree in Measuring and Control Technology and Instrumentations, the master's degree in Instrument Science and Technology from Southeast University in 2007 and 2010, and the doctoral degree in Informatics from Kyoto University in 2014. His research interests include computer vision, pattern recognition, and machine learning.

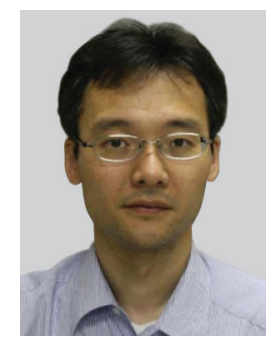

Masayuki Mukunoki received the bachelor's, the master's, and the doctoral degrees in Information Engineering from Kyoto University. He is now an Associate Professor in the Academic Center for Computing and Media Studies and a faculty member in the Graduate School of Informatics, in Kyoto University. His research interests include computer vision, video media processing, lecture video analysis and human activity sensing with camera.

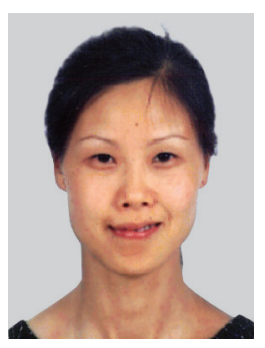

Yinghui Kuang is a professor and the Vice Dean of Chien-Shiung Wu College (Honors), Southeast University, China. She received the bachelor's degree in Mechanical Engineering and the master's degree in Automation in Machining from Xi' an Jiaotong University, and the doctoral degree in Measurement Technology and Instrumentation from Southeast University. Her research interests mainly include measurement and control technology and intelligent systems.

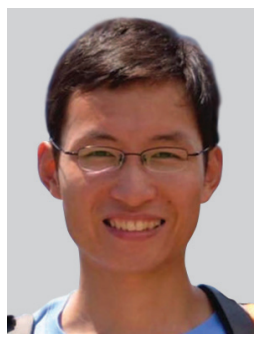

Yang Wu is currently a post-doctoral researcher of Academic Center for Computing and Media Studies, Kyoto University. He received a BS degree in information engineering and a $\mathrm{Ph} . \mathrm{D}$ degree in pattern recognition and intelligent systems from Xi' an Jiaotong University in 2004 and 2010, respectively. From Sept. 2007 to Dec. 2008, he was a visiting student in the General Robotics, Automation, Sensing and Perception (GRASP) lab at University of Pennsylvania. His research is in the fields of computer vision and pattern recognition, with particular interests in the detection, tracking and recognition of humans and also generic objects. He is also interested in image/video search and retrieval, along with machine learning techniques.

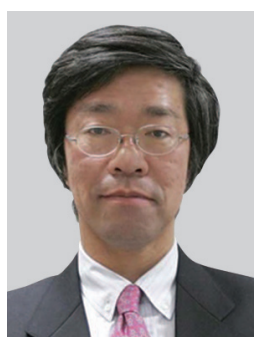

Michihiko Minoh is a professor at Academic Center for Computing and Media Studies (ACCMS), Kyoto University, Japan. He received the B. Eng., M. Eng., and D. Eng. degrees in Information Science from Kyoto University, in 1978, 1980 and 1983, respectively. He served as director of ACCMS from April 2006 to March 2010 and concurrently served as vice director in the Kyoto University Presidents Office from October 2008 to September 2010. Since October 2010, he has been vice-president, chief information officer at Kyoto University, and director-general at Institute for Information Management and Communication, Kyoto University. His research interests include a variety area of Image Processing, Artificial Intelligence and Multimedia Applications, particularly, model centered framework for the computer system to help visual communication among humans and information media structure for human communication. He is a member of Information Processing Society of Japan, Institute of Electronics, Information and Communication Engineers of Japan, the IEEE Computer Society and Communication Society, and ACM. 